Pacific Journal of Mathematic 


\section{ON $(\boldsymbol{J}, \boldsymbol{M}, \mathfrak{m})$-EXTENSIONS OF ORDER SUMS OF DISTRIBUTIVE LATTICES}

\section{RAYMOND BALBES}

In the first section of this paper a characterization of the order sum of a family $\left\{L_{\alpha}\right\}_{\alpha \in S}$ of distributive lattices is given which is analogous to the characterization of a free distributive lattice as one generated by an independent set. We then consider the collection $Q$ of order sums obtained by taking different partial orderings on $S$. A natural partial ordering is defined on $Q$ and its maximal and minimal elements are characterized.

Let $J$ and $M$ be collections of nonempty subsets of a distributive lattice $L$, and $m$ a cardinal. We define a $(J, M, \mathfrak{m})$ extension $(\psi, E)$ of $L$, where $E$ is a $\mathfrak{m}$-complete distributive lattice and $\psi: L \rightarrow E$ is a $(J, M)$-monomorphism. In the last section we define a m-order sum of a family of distributive lattices $\left\{L_{\alpha}\right\}_{\alpha \in S}$. The main result here is that the m-order sum exists if the order sum $L$ of $\left\{L_{\alpha}\right\}_{\alpha \in S}$ has a $(\boldsymbol{J}, \boldsymbol{M}, \mathfrak{m})$-extension, where $J$ and $M$ are certain collections of subsets of $L$. These results are analogous to $R$. Sikorski's work in Boolean algebras (e.g., [6]).

1. Order sums. Let $S$ be a fixed set and $\left\{L_{\alpha}\right\}_{\alpha \in S}$ a fixed collection of distributive lattices. From [2] it follows that for each poset $P=(S, \leqq)$, there exists a pair $\left(\left\{\varphi_{\alpha}\right\}_{\alpha \in S}, L(P)\right)$, where $L(P)$ is a distributive lattice, and for each $\alpha \in S, \varphi_{\alpha}: L_{\alpha} \rightarrow L(P)$ is a monomorphism such that:

(1.1) $L$ is generated by $\cup_{\alpha \in S} \varphi_{\alpha}\left(L_{\alpha}\right)$.

(1.2) If $\alpha<\beta$ then $\varphi_{\alpha}(x)<\varphi_{\beta}(y)$, for all $x \in L_{\alpha}$ and $y \in L_{\beta}$.

(1.3) If $\mathrm{M}$ is a distributive lattice and $\left\{f_{\alpha}: L_{\alpha} \rightarrow M\right\}_{\alpha \in S}$ is a family of homomorphisms such that $f_{\alpha}(x) \leqq f_{\beta}(y)$ whenever $\alpha<\beta$, $x \in L_{\alpha}$ and $y \in L_{\beta}$, then there exists a homomorphism $f: L(P) \rightarrow M$ such that $f \varphi_{\alpha}=f_{\alpha}$ for each $\alpha \in S$.

The pair $\left(\left\{\varphi_{\alpha}\right\}_{\alpha \in S}, L(P)\right)$ will be called an order sum of $\left\{L_{\alpha}\right\}_{\alpha \in S}$ over $P$.

Let $P$ be the family of all posets of the form $(S, \leqq)$ and let $Q=\left\{\left(\left\{\varphi_{\alpha}\right\}_{\alpha \in S}, L(P)\right) \mid P \in P\right\}$. For $\left(\left\{\varphi_{\alpha}\right\}_{\alpha \in S}, L(P)\right)$ and $\left(\left\{\theta_{\alpha}\right\}_{\alpha \in S}, L\left(P^{\prime}\right)\right)$ in $Q$ we write

(1.4) $\left(\left\{\varphi_{\alpha}\right\}_{\alpha \in S}, L(P)\right) \leqq\left(\left\{\theta_{\alpha}\right\}_{\alpha \in S}, L\left(P^{\prime}\right)\right)$ provided:

(1.5) there is a homomorphism $f: L\left(P^{\prime}\right) \rightarrow L(P)$ such that $f \theta_{\alpha}=$ $\varphi_{\alpha}$ for each $\alpha \in S$.

Note that (1.5) implies $f$ is an epimorphism. If $f$ is an isomor- 
phism, we say that $\left(\left\{\varphi_{\alpha}\right\}_{\alpha \in S}, L(P)\right)$ is isomorphic with $\left(\left\{\varphi_{\alpha}\right\}_{\alpha \in S}, L\left(P^{\prime}\right)\right)$. Isomorphism in this sense is an equivalence relation $\simeq$, and $[2, T h$. 1.2] implies that any two order sums over $P$ are isomorphic. By identifying isomorphs, (1.4) determines a partial ordering on the equivalence classes of $Q / \simeq$.

Definition 1.1. Suppose $P \in \boldsymbol{P}$ and $\left\{N_{\alpha}\right\}_{\alpha \in S}$ is a family of sublattices of a distributive lattices $N$. The family $\left\{N_{\alpha}\right\}_{\alpha \in S}$ is called $P$-independent if whenever $\alpha_{1}, \cdots, \alpha_{m}$ are distinct elements of $S, a_{m+1}, \cdots, \alpha_{n}$ are distinct elements of $S$ and $x_{i} \in N_{\alpha_{i}}$ for $i=1, \cdots, n$ then

(1.6) $x_{1} \cdot \cdots \cdot x_{m} \leqq x_{m+1}+\cdots+x_{n}$ if and only if

(1.7) for some $i$ and $j$, either $\alpha_{i}<\alpha_{j}$ or $\alpha_{i}=\alpha_{j}$ and $x_{i} \leqq x_{j}$, where $1 \leqq i \leqq m$ and $m+1 \leqq j \leqq n$.

Lemma 1.2. Suppose $N$ and $M$ are distributive lattices and $\left\{N_{\alpha}\right\}_{\alpha \in S}$ is a collection of sublattices of $N$ such that $\cup_{\alpha \in S} N_{\alpha}$ generates N. A necessary and sufficient condition for a family $\left\{f_{\alpha}: N_{\alpha} \rightarrow M\right\}_{\alpha \in S}$ of homomorphisms to have a common extension on $N$ is that if $\alpha_{1}, \cdots, \alpha_{m}$ are distinct members of $S, \alpha_{m+1}, \cdots, \alpha_{n}$ are distinct members of $S, x_{i} \in N_{\alpha_{i}}$ for $i=1, \cdots, n$ and

(1.8) $x_{1} \cdots \cdots x_{m} \leqq x_{m+1}+\cdots+x_{n}$ then

(1.9) $f_{\alpha_{1}}\left(x_{1}\right) \cdots \cdot f_{\alpha_{m}}\left(x_{m}\right) \leqq f_{\alpha_{m+1}}\left(x_{m+1}\right)+\cdots+f_{\alpha_{n}}\left(x_{n}\right)$.

Proof. The necessity is clear. Now if $x \in N_{\alpha} \cap N_{\beta}$ then by (1.9), $x \leqq x$ implies that $f_{\alpha}(x)=f_{\beta}(x)$. So the function $f: \cup_{\alpha \in S} N_{\alpha} \rightarrow M$ defined by $f(x)=f_{\alpha}(x)$ if $x \in L_{\alpha}$ makes sense and has the property that if $A$ and $B$ are finite nonempty subsets of $\cup_{\alpha \in \alpha S} N_{\alpha}$, then $\Pi_{N}(A) \leqq \Sigma_{N}(B)$ implies $\Pi_{M} f(A) \leqq \Sigma_{M} f(B)$. By [1, Lemma 1.7], $f$ can be extended to a homomorphism $f^{\prime}: N \rightarrow M$. This is the required extension.

THEOREM 1.3. The pair $\left(\left\{\theta_{\alpha}\right\}_{\alpha \in S}, L\right)$ is the order sum of $\left\{L_{\alpha}\right\}_{\alpha \in S}$ over $\boldsymbol{P} \in \boldsymbol{P}$ if and only if $\left\{\theta_{\alpha}: L_{\alpha} \rightarrow L\right\}_{\alpha \in S}$ is a family of monomorphisms such that:

(1.10) $\cup_{\alpha \in S} \theta_{\alpha}\left(L_{\alpha}\right)$ generates $L$, and

(1.11) $\left\{\theta_{\alpha}\left(L_{\alpha}\right)\right\}_{\alpha \in S}$ is P-independent.

Proof. For the sufficiency suppose first that $\alpha<\beta$. By (1.11) $\theta_{\alpha}(x) \leqq \theta_{\beta}(y)$ for all $x \in L_{\alpha}, y \in L_{\beta}$. But if $\theta_{\beta}(y) \leqq \theta_{\alpha}(x)$ then $\beta \leqq \alpha$. Hence (1.2) is satisfied. Now assume the hypothesis of (1.3). It is sufficient to show that the family $\left\{f_{\alpha} \theta_{\alpha}^{-1}: \theta_{\alpha}\left(L_{\alpha}\right) \rightarrow M\right\}_{\alpha \in S}$ has a common extension on $L$. So if

$$
\theta_{\alpha_{1}}\left(x_{1}\right) \cdot \cdots \cdot \theta_{\alpha_{m}}\left(x_{m}\right) \leqq \theta_{\alpha_{m+1}}\left(x_{m+1}\right)+\cdots+\theta_{\alpha_{n}}\left(x_{n}\right)
$$


where $\alpha_{1}, \cdots, \alpha_{m}$ are distinct and $\alpha_{m+1}, \cdots, \alpha_{n}$ are distinct then by (1.11) there exists $p, q$ such that $\alpha_{p}<\alpha_{q}$ or $\alpha_{p}=\alpha_{q}$ and $\theta_{\alpha_{p}}\left(x_{p}\right) \leqq \theta_{\alpha_{q}}\left(x_{q}\right)$, where $1 \leqq p \leqq m$ and $m+1 \leqq q \leqq n$. In any case $f_{\alpha_{p}}\left(x_{p}\right) \leqq f_{\alpha_{q}}\left(x_{q}\right)$ and so

$$
\prod_{i=1}^{m} f_{\alpha_{i}} \theta_{\alpha_{i}}^{-1} \theta_{\alpha_{i}}\left(x_{i}\right) \leqq \sum_{\jmath=m+1}^{n} f_{\alpha_{j}} \theta_{\alpha_{j}}^{-1} \theta_{\alpha_{j}}\left(x_{j}\right) .
$$

The result now follows from Lemma 1.2. The converse is essentially [2, Th. 1.9].

The set $\boldsymbol{P}$ can be partially ordered as follows. If $P, P^{\prime} \in \boldsymbol{P}$ then $P \leqq P^{\prime}$ provided $P^{\prime} \cong P$, as sets of ordered pairs. It is immediate that $\boldsymbol{P}$ has a greatest element-the trivial partial ordering on $S$. Also, it can be shown that $P$ is minimal in $P$ if and only if $P$ is a chain.

\section{THEOREM 1.4. $P \cong Q / \simeq$.}

Proof. It is sufficient to show that for $\left(\left\{\varphi_{\alpha}\right\}_{\alpha \in S}, L(P)\right),\left(\left\{\theta_{\alpha}\right\}_{\alpha \in S}\right.$, $\left.L\left(P^{\prime}\right)\right) \in Q$ :

$$
\text { (1.12) } P \leqq P^{\prime}
$$

if and only if

(1.13) $\quad\left(\left\{\varphi_{\alpha}\right\}_{\alpha \in S}, L(P)\right) \leqq\left(\left\{\theta_{\alpha}\right\}_{\alpha \in S}, L\left(P^{\prime}\right)\right)$.

If $P \leqq P^{\prime}$, then $\left\{\varphi_{\alpha}: L_{\alpha} \rightarrow L(P)\right\}_{\alpha \in S}$ is a family of homorphisms with the property that if $\alpha<\beta$ (in $P^{\prime}$ ) then $\varphi_{\alpha}(x)<\varphi_{\beta}(y)$ for all $x \in L_{\alpha}$, $y \in L_{\beta}$. So by (1.3), we have (1.13). Conversely, suppose (1.5) holds and $\alpha<\beta$ (in $P^{\prime}$ ). Letting $x \in L_{\alpha}$ and $y \in L_{\beta}$, we have $\theta_{\alpha}(x)<\theta_{\beta}(y)$ so $\varphi_{\alpha}(x)=f \theta_{\alpha}(x) \leqq f \theta_{\beta}(y)=\varphi_{\beta}(y)$. Since $\left\{\varphi_{\alpha}\left(L_{\alpha}\right)\right\}_{\alpha \in S}$ is $P$-independent, $\alpha \leqq \beta$ (in $P$ ). It follows that $P^{\prime} \subseteq P$.

CoRollary 1.5. $\left(\left\{\varphi_{\alpha}\right\}_{\alpha \in S}, L(P)\right) / \simeq$ is the greatest element in $Q / \simeq$ if and only if $L(P)$ is the free product of $\left\{L_{\alpha}\right\}_{\alpha \in S}$. Furthermore, $\left(\left\{\varphi_{\alpha}\right\}_{\alpha \in S}, L(P)\right) / \simeq$ is minimal in $Q / \simeq$ if and only if $L(P)$ is an ordinal sum of $\left\{L_{\alpha}\right\}_{\alpha \in S}$.

Proof. The definitions of free product and ordinal sum can be found in [7, §9] and [2, Definition 1.3]. The result then follows from Theorem 1.4 and the remark following Theorem 1.3.

For the remainder of this section, let $\left(\left\{\varphi_{\alpha}\right\}_{\alpha \in S}, L(P)\right)$ be a fixed member of $Q$.

A lattice $L$ is said to be conditionally implicative if for each pair $x, y \in L$ such that $x \notin y$ there is an element $x \rightarrow y$ with the property that $x \cdot z \leqq y$ if and only if $z \leqq x \rightarrow y$. Note that conditionally 
implicative lattices are distributive. The following theorem, which we stated without proof in [2], is the converse of [2, Th. 2.5].

THEOREM 1.6. If $L(P)$ is conditionally implicative then $L_{\alpha}$ is conditionally implicative for each $\alpha \in S$.

Proof. Let $x, y \in L_{\alpha}$ and $x \not y$. Then $\varphi_{\alpha}(x) \rightarrow \varphi_{\alpha}(y)$ exists in $L(P)$ and equals a sum of $m$ products, each of the form

$$
\varphi_{\gamma_{1}}\left(x_{1}\right) \cdots \cdots \varphi_{\gamma_{n}}\left(x_{n}\right) \text {. }
$$

We can assume $\gamma_{i} \not \equiv \gamma_{j}$ for $i \neq j$. Now

$$
\varphi_{\alpha}(x)\left(\varphi_{r_{1}}\left(x_{1}\right) \cdots \cdots \varphi_{r_{n}}\left(x_{n}\right)\right) \leqq \varphi_{\alpha}(x)\left(\varphi_{\alpha}(x) \rightarrow \varphi_{\alpha}(y)\right) \leqq \varphi_{\alpha}(y) .
$$

By (1.11) there exists $p$ such that $\gamma_{p}<\alpha$ or $\gamma_{p}=\alpha$ and $x x_{p} \leqq y$. But in any case $\varphi_{\alpha}(x) \varphi_{\gamma_{p}}\left(x_{p}\right) \leqq \varphi_{\alpha}(y)$. Hence

(1.14) $\varphi_{r_{p}}\left(x_{p}\right) \leqq \varphi_{\alpha}(x) \rightarrow \varphi_{\alpha}(y)$.

Choosing an element $\varphi_{\beta_{j}}\left(y_{j}\right)$, that satisfies (1.14), from each of the $m$ summands of $\varphi_{\alpha}(x) \rightarrow \varphi_{\alpha}(y)$, we have:

$$
\sum_{j=1}^{m} \varphi_{\beta_{j}}\left(y_{j}\right) \leqq \varphi_{\alpha}(x) \rightarrow \varphi_{\alpha}(y) \leqq \sum_{j=1}^{m} \phi_{\beta_{j}}\left(y_{j}\right) \text {, }
$$

and so $\varphi_{\alpha}(x) \rightarrow \varphi_{\alpha}(y)=\sum_{j=1}^{p} \varphi_{\beta_{j}}\left(y_{j}\right)$, where $\beta_{i} \not \geqq \beta_{j}$ for $i \neq j$. For each $j, \varphi_{\alpha}(x) \varphi_{\beta_{j}}\left(y_{j}\right) \leqq \varphi_{\alpha}(x)\left(\varphi_{\alpha}(x) \rightarrow \varphi_{\alpha}(y)\right) \leqq \varphi_{\alpha}(y)$, and since $x \leqq y$, we have: $\beta_{j} \leqq \alpha$ for $j=1, \cdots, p$. But $\varphi_{\alpha}(y) \leqq \varphi_{\alpha}(x) \rightarrow \varphi_{\alpha}(y)=\varphi_{\beta_{1}}\left(y_{1}\right)+\cdots+$ $\varphi_{\beta_{p}}\left(y_{p}\right)$. Hence there exists $j_{0}$ such that $\alpha \leqq \beta_{j_{0}}$. Since $\alpha=\beta_{j_{0}}$ and $\alpha>\beta_{j}$ for $j \neq j_{0}$, we have $\varphi_{\alpha}(x) \rightarrow \varphi_{\alpha}(y)=\varphi_{\alpha}\left(x_{j_{0}}\right)$. From the fact that $\varphi_{\alpha}$ is a monomorphism, it is now easy to show that $x \rightarrow y=x_{j_{0}}$.

The following property of $\varphi_{\alpha}$ will be needed in $\S 3$. Note that the power of a set $H$ is denoted by $|H|$.

Definition 1.7. Let $L$ and $M$ be distributive lattices and $m$ a cardinal. A homomorphism $h: L \rightarrow M$ is called a m-homomorphism provided:

If $H \subseteq L, 0<|H| \leqq \mathfrak{m}$, and $\Sigma_{L}(H)$ exists then $\Sigma_{M} h(H)$ exists and equals $h\left(\Sigma_{L}(H)\right)$; and similarly for products. The homomorphism is complete if it is a $\mathrm{m}$-homomorphism for each cardinal $\mathrm{m}$.

LEMMA 1.8. Each monomorphism $\varphi_{\alpha}: L_{\alpha} \rightarrow L(P)$ of $\left(\left\{\varphi_{\alpha}\right\}_{\alpha \in S}, L(P)\right)$ is complete.

Proof. Let $H \cong L_{\alpha}$ and suppose $x=\Sigma_{L_{\alpha}}(H)$ exists. Clearly $\varphi_{\alpha}(y) \leqq \varphi_{\alpha}(x)$ for all $y \in H$. Now suppose that $\Sigma_{L(P)}\left(H_{1}\right) \cdots \cdots \Sigma_{L(P)}\left(H_{n}\right)$ is an upper bound for $\varphi_{\alpha}(H)$, where $H_{i} \subseteq \cup_{\alpha \in S} \varphi_{\alpha}\left(L_{\alpha}\right)$ for $i=1, \cdots, n$. 
We can assume $H_{1}=\left\{\varphi_{\alpha_{1}}\left(x_{1} \cdots \cdots \varphi_{\alpha_{m}}\left(x_{m}\right)\right\}\right.$ where $x_{i} \in L_{\alpha_{i}}$ and $\alpha_{k} \neq \alpha_{j}$ for $k \neq j$. Suppose:

(1.15) there exists $j \in\{1, \cdots, m\}$ such that $\alpha<\alpha_{j}$. Then $\varphi_{\alpha}(x)<\varphi_{\alpha_{j}}\left(x_{j}\right)$ so

(1.16) $\varphi_{\alpha}(x) \leqq \Sigma_{L(P)}\left(H_{1}\right)$.

Now suppose that (1.15) does not hold. Since $\varphi_{\alpha}(y) \leqq \varphi_{\alpha_{1}}\left(x_{1}\right)+\cdots+$ $\varphi_{\alpha_{m}}\left(x_{m}\right)$ for each $y \in H$, and $\alpha_{j} \neq \alpha_{k}$ for $j \neq k$, there exists $\alpha_{j}$ such that $\alpha=\alpha_{j}$ and $\varphi_{\alpha}(y) \leqq \varphi_{\alpha_{j}}\left(x_{j}\right)$ for all $y \in H$. Hence $x_{j} \in L_{\alpha}$ and $y \leqq x_{j}$ for all $y \in S$. So $x \leqq x_{j}$ and therefore (1.16) is valid regardless of the validity of (1.15). Applying this argument to each $H_{i}$, we have $\varphi_{\alpha}(x) \leqq \Sigma_{L(P)}\left(H_{1}\right) \cdots \cdots \Sigma_{L(P)}\left(H_{n}\right)$, and so $\varphi_{\alpha}\left(\Sigma_{L_{\alpha}}(H)\right)=\Sigma_{L(P)} \varphi_{\alpha}(H)$. Similarly for products.

2. $(J, M, \mathfrak{m})$-extensions. Throughout this section, let $L$ be a distributive lattice, and $\mathfrak{m}$ a fixed infinite cardinal. Also let $J$ and $M$ be collections of nonempty subsets of $L$ such that

(2.1) $|H| \leqq \mathfrak{m}$ for each $H \in J$ and each $H \in M$.

(2.2) $\Sigma_{L}(H)$ exists for each $H \in J$ and $\Pi_{L}(H)$ exists for each $H \in M$.

Definition 2.1. If $L^{\prime}$ is a distributive lattice then a homomorphism $f: L \rightarrow L^{\prime}$ is a $(\boldsymbol{J}, \boldsymbol{M})$-homomorphism provided:

(2.3) If $H \in J$ then $\Sigma_{L^{\prime}} f(H)$ exists and equals $f\left(\Sigma_{L}(H)\right)$.

(2.4) If $H \in M$ then $\Pi_{L^{\prime}} f(H)$ exists and equals $f\left(\Pi_{L}(H)\right)$.

Definition 2.2. The pair $(\psi, E)$ is called a $(\boldsymbol{J}, \boldsymbol{M}, \mathfrak{m})$-extension of $L$ provided:

(2.5) $E$ is a m-complete distributive lattice.

(2.6) $\psi: L \rightarrow E$ is a $(J, M)$-monomorphism.

(2.7) $\psi(L)$ m-generates $E$ (i.e., $E$ is the smallest nt-complete sublattice of $E$ that contains $\psi(L))$.

Every distributive lattice has a $(\dot{\phi}, \dot{\phi}, \mathfrak{m})$-extension: the smallest m-ring of subsets of the Stone space $X$ of $L$ that contains all of the compact-open sets of $X$, together with the correspondence that associates elements of $L$ with compact-open sets of $X$. If $\boldsymbol{J}(\boldsymbol{M})$ is the collection of all subsets of $L$ of power $\leqq m$ which have a sum (product) in $L$ then a $(\boldsymbol{J}, \boldsymbol{M}, \mathfrak{m})$-extension of $L$ is called a $\mathfrak{m}$-regular extension. Note that in this case, $\psi$ is a m-homomorphism. In [5], Crawley has constructed an example of a distributive lattice which can not be regularly imbedded in any complete distributive lattice. In this example if we take $I$ to be countable then $L$ will have no $\boldsymbol{\aleph}_{0}$-regular extension.

A sufficient condition for $L$ to have a $(\boldsymbol{J}, \boldsymbol{M}, \mathrm{m})$-extension is that $L$ be conditionally implicative. Indeed, it is easily verified that the MacNeille completion [3, p. 58] of such a lattice is also conditionally implicative and hence distributive. Note that the category of condi- 
tionally implicative lattices includes the categories of Boolean algebras, chains, free and finite distributive lattices, and pseudo Boolean algebras. Another sufficient condition for $L$ to have a $(J, M, \mathfrak{m})$-extension is that

$$
y \sum_{i \in I} x_{i}=\sum_{i \in I} y x_{i} \text { and } y+\prod_{i \in I} x_{i}=\prod_{i \in I}\left(y+x_{i}\right)
$$

whenever the left sides exist and $|I| \leqq \mathfrak{m}$. This follows from [4, Lemma 2]. write

If $(\psi, E)$ and $\left(\psi^{\prime}, E^{\prime}\right)$ are $(J, M, \mathfrak{m})$-extensions of $L$, then we

$$
\text { (2.9) }(\psi, E) \leqq\left(\psi^{\prime}, E^{\prime}\right)
$$

provided there is a m-homomorphism $h: E^{\prime} \rightarrow E$ such that $h \psi^{\prime}=\psi$. Clearly $h$ is onto. If $h$ is an isomorphism we say $(\psi, E)$ is isomorphic with $\left(\psi^{\prime}, E^{\prime}\right)$. Isomorphism in this sense is an equivalence relation $\simeq$, and by identifying isomorphs, (2.9) determines a partial ordering on the equivalence classes of $K / \simeq$ where $K$ is the set of $(J, M, \mathfrak{m})$ extensions of $L$.

By generalizing the method in $[6, p .166]$, we now investigate the class $K$.

DEFINITION 2.3. A congruence relation $R$ on a m-complete lattice $M$ is called a m-congruence relation on $M$ if whenever $I$ is an index set of power $\leqq \mathfrak{M}$ and $\left(x_{i}, y_{i}\right) \in R$ for each $i \in I$ then

$$
\left(\Sigma\left\{x_{i} \mid i \in I\right\}, \Sigma\left\{y_{i} \mid i \in I\right\}\right) \in R \text { and }\left(\Pi\left\{x_{i} \mid i \in I\right\}, \Pi\left\{y_{i} \mid i \in I\right\}\right) \in R .
$$

For a m-congruence relation $R$ on a m-complete lattice $M$, let $[x]_{R}$ be the equivalence class containing $x \in M$, and let

$$
M / R=\{[x] \mid x \in M\} .
$$

The following theorem is easily verified.

THEOREM 2.4. If $R$ is a m-congruence relation on a m-complete lattice $M$ then $M / R$ is partially ordered as follows: $[x]_{R} \leqq[y]_{R}$ provided there exists $x^{\prime}, y^{\prime} \in M$ such that $\left(x, x^{\prime}\right) \in R, x^{\prime} \leqq y^{\prime}$ and $\left(y^{\prime}, y\right) \in R$. Furthermore, $M / R$ is a m-complete lattice such that if $H \leqq M \quad$ and $\quad 0<|H| \leqq \mathfrak{m}$ then $\Sigma_{M / R}\left\{[x]_{R} \mid x \in H\right\}=\left[\Sigma_{M}(H)\right]_{R}$ and $\Pi_{M / R}\left\{[x]_{R} \mid x \in H\right\}=\left[\Pi_{M}(H)\right]_{R}$. If $M$ is distributive so is $M / R$.

Let $\mathfrak{n}$ be the power of the distributive lattice $L$ and let $F$ be the free $\mathfrak{m}$-complete distributive lattice with $\mathfrak{n}$ generators. That is, $F$ satisfies:

(2.10) $F$ is a m-complete distributive lattice and is $\mathfrak{m}$-generated by a subset $G$ of power $\pi$.

(2.11) If $h: G \rightarrow M$ is a function, where $M$ is a m-complete 
distributive lattice, then $h$ can be extended to a m-homomorphism on $F$.

By (2.11), $G$ has the property that if $G_{1}, G_{2}$ are finite nonempty subsets of $G$ and $\Pi_{F}\left(G_{1}\right) \leqq \Sigma_{F}\left(G_{2}\right)$, then $G_{1} \cap G_{2} \neq \phi$. So the sublattice $F^{\prime}$ generated by $G$ is freely generated by $G$, and there is an epimorphism $g: F^{\prime} \rightarrow L$. Let $R$ be the set of m-congruence relations $R$ on $F$ such that:

(2.12) If $x, y \in F^{\prime}$ then $(x, y) \in R \Leftrightarrow g(x)=g(y)$.

(2.13) If $H \subseteq F^{\prime},|H| \leqq \mathfrak{m}, g(H) \in J, x \in F^{\prime}$, and $g(x)=\Sigma_{L} g(H)$ then $\left(x, \Sigma_{F}(H)\right) \in R$.

(2.14) If $H \subseteq F^{\prime},|H| \leqq \mathfrak{m}, g(H) \in M, x \in F^{\prime}$ and $g(x)=\Pi_{L} g(H)$ then $\left(x, \Pi_{F}(H)\right) \in R$.

For each $R \in \boldsymbol{R}$, let $F_{R}^{\prime}$ be the sublattice $\left\{[x]_{R} \mid x \in F^{\prime}\right\}$ of $F / R$. By (2.12), the mapping $g_{R}: F_{R}^{\prime} \rightarrow L$ defined by:

(2.15) $g_{R}\left([x]_{R}\right)=g(x)$ for each $x \in F^{\prime}$ is an isomorphism. Define $\psi_{R}: L \rightarrow F / R$ by $\psi_{R}=i_{R} g_{R}^{-1}$ where $i_{R}: F_{R}^{\prime} \rightarrow F / R$ is the inclusion map. We have

(2.16) $\psi_{R} g(x)=[x]_{R}$ for each $x \in F^{\prime}$.

Theorem 2.5. For each $R \in \boldsymbol{R}$, the pair $\left(\psi_{R}, F / R\right)$ is a $(\boldsymbol{J}, \boldsymbol{M}, \mathfrak{m})$ extension of $L$.

Proof. First $F / R$ is m-complete by Theorem 2.4. Let $G \in J$, then $|G| \leqq \mathfrak{m}$ and $\Sigma_{L}(G)$ exists. Since $g$ is onto $L$ there exists $\{x\} \cup H \subseteq F^{\prime}$ such that $|H| \leqq \mathfrak{m}, \quad g(H)=G$ and $g(x)=\Sigma_{L} g(H)$. By (2.13), $\left(x, \Sigma_{F}(H)\right) \in \boldsymbol{R}$ so

$$
\begin{aligned}
\psi_{R}\left(\Sigma_{L}(G)\right) & =[x]_{R}=\left[\Sigma_{F}(H)\right]_{R}=\Sigma_{F / R}\left\{[y]_{R} \mid y \in H\right\} \\
& =\Sigma_{F / R} \psi_{R} g(H)=\Sigma_{F / R} \psi_{R}(G) .
\end{aligned}
$$

A similar argument for $G \in \boldsymbol{M}$ implies that $\psi_{R}$ is a $(\boldsymbol{J}, \boldsymbol{M})$-monomorphism. Finally since

$$
\psi_{R}(L)=\psi_{R} g\left(F^{\prime}\right)=F_{R}^{\prime}
$$

and $F^{\prime}$ m-generates $F$, we have $\psi_{R}(L)$ m-generates $F / R$.

THEOREM 2.6. For each $(J, M, \mathfrak{m})$-extension $(\psi, E)$ of $L$, there exists $R \in \boldsymbol{R}$ such that $(\psi, E) \simeq\left(\psi_{R}, F / R\right)$.

Proof. By (2.11), the mapping $\psi g: F^{\prime} \rightarrow E$ can be extended to a m-homomorphism $k$ of $F$ onto $E$. Define a relation $R$ on $F$ by $(x, y) \in R$ if $k(x)=k(y)$. It is easily verified that $R \in R$ so that by Theorem 2.5, $\left(\psi_{R}, F / R\right)$ is a $(J, M, \mathfrak{m})$-extension of $L$. Next, define $h: F / R \rightarrow E$ by $h\left([x]_{R}\right)=k(x)$ for each $x \in F$. Then $h$ is an isomorphism. Let $y \in L$, then there is an $x \in F^{\prime}$ such that $g(x)=y$, so 


$$
h \psi_{R}(y)=h \psi_{R} g(x)=h\left([x]_{R}\right)=k(x)=\psi g(x)=\psi(y) .
$$

It follows that $(\psi, E) \simeq\left(\psi_{R}, F / R\right)$.

THEOREM 2.7. If $\left(\psi_{R}, F / R\right)$ and $\left(\psi_{R^{\prime}}, F / R^{\prime}\right)$ are $(\boldsymbol{J}, M, \mathfrak{m})$-extensions of $L$ then

$$
\left(\psi_{R}, F / R\right) \leqq\left(\psi_{R^{\prime}}, F / R^{\prime}\right)
$$

if and only if

$$
R^{\prime} \cong R
$$

Consequently, $K / \simeq$ is isomorphic with $\boldsymbol{R}$ (partially ordered by the converse of inclusion).

Proof. Suppose there is a m-epimorphism $h: F / R^{\prime} \rightarrow F / R$ such that $h \psi_{R^{\prime}}=\psi_{R}$. For each $\left.x \in F^{\prime}, h([x)]_{R^{\prime}}\right)=h \psi_{R^{\prime}} g(x)=\psi_{R} g(x)=[x]_{R}$. But, in fact, $\left\{x \in F \mid h\left([x]_{R^{\prime}}\right)=[x]_{R}\right\}$ is a m-sublattice of $F$ containing $F^{\prime}$. So $h\left([x]_{R^{\prime}}\right)=[x]_{R}$ for each $x \in F$. Thus if $(x, y) \in R^{\prime}$ then $[x]_{R}=$ $h\left([x]_{R^{\prime}}\right)=h\left([y]_{R^{\prime}}=[y]_{R}\right.$, i.e., $R^{\prime} \subseteq R$. For the converse, define $h: F / R^{\prime}$ $\rightarrow F / R$ by $h\left([x]_{R^{\prime}}\right)=[x]_{R}$ for each $x \in F$. The hypothesis implies $h$ is a m-homomorphism. Since $h \psi_{R^{\prime}}=\psi_{R}$, the result follows.

COROLLARY 2.8. The intersection $\rho=\bigcap_{R \in R} R$ is an element of $\boldsymbol{R}$ and hence the equivalence class containing $\left(\psi_{\rho}, F / \rho\right)$ is the greatest element in $K / \simeq$. Here it is ass'urned $\boldsymbol{R} \neq \varnothing$.

Proof. Conditions (2.12), (2.13), and (2.14) are satisfied by $\rho$.

Definition 2.9. A $(\boldsymbol{J}, \boldsymbol{M}, \mathrm{m})$-extension $(\psi, E)$ of $L$ is said to be free provided that for each m-complete distributive lattice $L^{\prime}$ and each $(\boldsymbol{J}, \boldsymbol{M})$-homomorphism $f: L \rightarrow L^{\prime}$, there exists a m-homomorphism $h: E \rightarrow L^{\prime}$ such that $f=h \psi^{\prime}$.

The main result of this section is then:

TheOREM 2.10. If $L$ has a $(\boldsymbol{J}, \boldsymbol{M}, \mathfrak{m})$-extension then $L$ has a free $(J, M, \mathfrak{m})$-extension: $\left(\psi_{\rho}, F / \rho\right)$.

Proof. As in the proof of Theorem 2.6, the mapping $f g: F^{\prime} \rightarrow L^{\prime}$ can be extended to a $\mathrm{m}$-homomorphism $h^{\prime}: F \rightarrow L^{\prime}$. Define a relation $R^{\prime}$ on $F$ by $(x, y) \in R^{\prime}$ if $h^{\prime}(x)=h^{\prime}(y)$. We first show that $R^{\prime} \cap \rho \in R$. Clearly $R^{\prime} \cap \rho$ is a m-congruence relation. For (2.12), (2.13), and (2.14), first let $x, y \in F^{\prime}$. Since $\rho \in R, \quad(x, y) \in R^{\prime} \cap \rho$ implies $g(x)=g(y)$. Conversely if $g(x)=g(y)$ then $f g(x)=f g(y)$ so $(x, y) \in \rho \cap R^{\prime}$. If 
$H \subseteq F^{\prime},|H| \leqq \mathfrak{m}, g(H) \in J, x \in F^{\prime}$ and $g(x)=\Sigma_{L} g(H)$ then since $\rho \in R$, $\left(x, \Sigma_{F}(H)\right) \in \rho$. But $f$ is a $(J, M)$-homomorphism so $f g(x)=f\left(\Sigma_{L} g(H)\right)=$ $\Sigma_{L^{\prime}} f g(H)$. Hence $h^{\prime}(x)=\Sigma_{L}, h^{\prime}(H)=h^{\prime}\left(\Sigma_{F}(H)\right)$, i.e., $\left(x, \Sigma_{F}(H)\right) \in \rho \cap R^{\prime}$. Similarly for (2.14). Now $\rho \cap R^{\prime} \in R$ so $\rho \subseteq R^{\prime}$. Hence we can define $h: F / \rho \rightarrow L^{\prime}$ by $h\left([x]_{\rho}\right)=h^{\prime}(x)$ for each $x \in F$. It follows that $h$ is a m-homomorphism and $f=h \psi_{\rho}$.

3. m-order sums. In this section $\left\{L_{\alpha}\right\}_{\alpha \in S}$ is a fixed set of distributive lattices, $\mathfrak{n}$ is a fixed infinite cardinal and $P$ is a partial ordering on $S$.

DEFINITION 3.1. The pair $\left(\left\{\psi_{\alpha}\right\}_{\alpha \in S}, E\right)$ is said to be a m-order sum of $\left\{L_{\alpha}\right\}_{\alpha \in S}$ over $P$ provided $E$ is a m-complete distributive lattice, and for each $\alpha \in S, \psi_{\alpha}: L_{\alpha} \rightarrow E$ is a m-monomorphism such that:

(3.1) $E$ is $\mathfrak{m}$-generated by $\bigcup_{\alpha \in S} \psi\left(L_{\alpha}\right)$.

(3.2) If $\alpha<\beta$ then $\psi_{\alpha}(x)<\psi_{\beta}(y)$ for each $x \in L_{\alpha}$ and $y \in L_{\beta}$.

(3.3) If $L^{\prime}$ is a m-complete distributive lattice and $\left\{f_{\alpha}: L_{\alpha} \rightarrow L^{\prime}\right\}_{\alpha \in S}$ is a collection of $m$-homomorphisms such that whenever $\alpha<\beta$ then $f_{\alpha}(x) \leqq f_{\beta}(y)$ for all $x \in L_{\alpha}, y \in L_{\beta}$, then there exists a nt-homomorphism $f: E \rightarrow L^{\prime}$ such that $f \psi_{\alpha}=f_{\alpha}$ for each $\alpha \in S$.

It follows that the m-order sum is essentially unique-if it exists. Note also that if $P$ is the trivial ordering on $S$ and $\left|L_{\alpha}\right|=1$ for each $\alpha \in S$ then $E$ is the free nt-complete distributive lattice with $|S|$ generators. We now investigate the existence question.

Let $\left(\left\{\varphi_{\alpha}\right\}_{\alpha \in S}, L(P)\right)$ be the order sum of $\left\{L_{\alpha}\right\}_{\alpha \in S}$ over $P$. Let $J$ be the class of all sets of the form $\varphi_{\alpha}(H)$ where

(3.4) $\alpha \in S, H \subseteq L_{\alpha},|H| \leqq \mathfrak{m}, H \neq \phi$

and such that $\Sigma_{L_{\alpha}}(H)$ exists. Let $M$ be the class of all sets of the form $\varphi_{\alpha}(H)$ satisfying (3.4) and such that $I_{L_{\alpha}}(H)$ exists. Note that since $\varphi_{\alpha}$ is a complete monomorphism (Lemma 1.8), conditions (2.1) and (2.2) of $\S 2$ are satisfied.

THEOREM 3.2. If $L(P)$ has a $(\boldsymbol{J}, \boldsymbol{M}, \mathrm{m})$-extension then $\left\{L_{\alpha}\right\}_{\alpha \in S}$ has a m-order sum over $P$.

Proof. By Theorem 2.10, $L(P)$ has a free $(\boldsymbol{J}, \boldsymbol{M}, \mathfrak{m})$-extension $(\psi, E)$. We. will show that $\left(\left\{\psi \varphi_{\alpha}\right\}_{\alpha \in S}, E\right)$ is the required m-order sum. Let $H \subseteq L_{\alpha}, 0<|H| \leqq \mathfrak{m}$ and suppose that $\Sigma_{L_{\alpha}}(H)$ exists. Then $\varphi_{\alpha}(H) \in J$. Since $\psi$ is a $(\boldsymbol{J}, \boldsymbol{M})$-monomorphism and $\varphi_{\alpha}$ is complete,

$$
\psi \varphi_{\alpha}\left(\Sigma_{L_{\alpha}}(H)\right)=\Sigma_{E} \psi \varphi_{\alpha}(H)
$$

Similary for products. So $\psi \varphi_{\alpha}$ is a m-monomorphism. Since $\mathrm{U}_{\alpha \in S}$ $\varphi_{\alpha}\left(L_{\alpha}\right)$ generates $L(P)$ and $\psi(L(P))$ m-generates $E$, it follows that 
$\bigcup_{\alpha \in S} \psi \varphi_{\alpha}\left(L_{\alpha}\right)$ m-generates $E$. Finally, let $L^{\prime}$ be a m-complete distributive lattice and $\left\{f_{\alpha}: L_{\alpha} \rightarrow L^{\prime}\right\}_{\alpha \in S}$ a family of m-homomorphisms with the property that $\alpha<\beta$ implies $f_{\alpha}(x) \leqq f_{\beta}(y)$ for all $x \in L_{\alpha}, y \in L_{\beta}$. By (1.3) three exists a homomorphism $f^{\prime}: L(P) \rightarrow L^{\prime}$ such that $f^{\prime} \varphi_{\alpha}=$ $f_{\alpha}$ for each $\alpha \in S$. Since $\varphi_{\alpha}$ is complete, $f^{\prime}$ is a $(\boldsymbol{J}, \boldsymbol{M})$-homomorphism. But $(\psi, E)$ is a free- $(\boldsymbol{J}, \boldsymbol{M})$-extension, so there exists a $\mathfrak{m}$-homomorphism $f: E \rightarrow L^{\prime}$ such that $f^{\prime}=f \psi$. Thus $f \psi \varphi_{\alpha}=f_{\alpha}$ for each $\alpha \in S$.

CoROLlary 3.3. If $\left\{L_{\alpha}\right\}_{\alpha \in S}$ is a collection of conditionally implicative lattices (or lattices satisfying (2.8)), then $\left\{L_{\alpha}\right\}_{\alpha \in S}$ has a morder sum over $P$ for each partial ordering $P$ on $S$.

Proof. This is immediate from Theorem 3.2 and the remarks following Definition 2.2.

A necessary condition for the m-order sum $\left(\left\{\psi_{\alpha}\right\}_{\alpha \in S}, E\right)$ over $P$ of $\left\{L_{\alpha}\right\}_{\alpha \in S}$ to exist is that each $L_{\alpha}$ have a free m-regular extension (consider the smallest $m$-complete sublattice of $E$ that contains $\psi_{\alpha}\left(L_{\alpha}\right)$ ). A case in which an m-order sum has a rather simple structure is obtained in the next theorem. For the definition of ordinal sum, see [2, Definition 1.3].

Theorem 3.4. Suppose $S$ is finite and $P$ is a chain in $P$. If $\left(\psi_{\alpha}, E_{\alpha}\right)$ is a free $\mathfrak{m}$-regular extension of $L_{\alpha}$ for each $\alpha \in S$, then $\left(\left\{i_{\alpha} \psi_{\alpha}\right\}_{\alpha \in S}, E\right)$ is the m-order sum of $\left\{L_{\alpha}\right\}_{\alpha \in S}$ over $P$, where $E$ is the ordinal sum of $\left\{E_{\alpha}\right\}_{\alpha \in S}$ and $i_{\alpha}: E_{\alpha} \rightarrow E$ is the inclusion map for each $\alpha \in S$.

Proof. We can assume that $S=\{1,2, \cdots, n\}$ with the usual ordering and $\left\{E_{\alpha}\right\}_{\alpha \in S}$ is a pair-wise disjoint family. Clearly, for $\mathrm{H} \subseteq E$, $0<|H|<\mathfrak{m}$, we have $\Sigma_{E}(H)=\Sigma_{E_{\beta}}\left(H \cap E_{\beta}\right)$ where $\beta=\max \{\alpha \in S \mid$ $\left.H \cap E_{\alpha} \neq \phi\right\}$. It is evident that $E$ is a m-complete distributive lattice, m-generated by $\bigcup_{\alpha \in S} i_{\alpha} \psi_{\alpha}\left(L_{\alpha}\right)$. Now assume the hypothesis of (3.3). Since $\left(\psi_{\alpha}, E_{\alpha}\right)$ is a $\mathfrak{m}$-regular extension of $L_{\alpha}$, there exists a m-homomorphism $g_{\alpha}: E_{\alpha} \rightarrow L^{\prime}$ such that $g_{\alpha} \psi_{\alpha}=f_{\alpha}$ for each $\alpha \in S$. The function $g: E \rightarrow L^{\prime}$ defined by $g(x)=g_{\alpha}(x)$ for $x \in E_{\alpha}$ has the property $g \psi_{\alpha}=f_{\alpha}$ for each $\alpha \in S$. To show $g$ preserves order, suppose $\alpha<\beta, x$ is a fixed element in $L_{\alpha}$ and let $F=\left\{y \in E_{\beta} \mid g_{\alpha} \psi_{\alpha}(x) \leqq g_{\beta}(y)\right\}$. Then

(i ) $\psi_{\beta}\left(L_{\beta}\right) \subseteq F$ and

(ii) $F$ is a m-complete sublattice of $E_{\beta}$.

It follows that $F=E_{\beta}$ and

$$
g_{\alpha} \psi_{\alpha}(x) \leqq g_{\beta}(y) \text { for } \quad x \in L_{\alpha}, y \in E_{\beta} .
$$


Now let $y$ be a fixed element of $E_{\beta}$ and let $G=\left\{z \in E_{\alpha} \mid g_{\alpha}(z) \leqq g_{\beta}(y)\right\}$. Then

(iii) $\psi_{\alpha}\left(L_{\alpha}\right) \subseteq G$ and

(iv) $G$ is a m-complete sublattice of $E_{\alpha}$.

It follows that $G=E_{\alpha}$ and that for $x \in L_{\alpha}, y \in L_{\beta}, g(x) \leqq g(y)$. Finally, to show $g$ is a $\mathfrak{m}$-homomorphism, let $H \subseteq E, 0<|H|<\mathfrak{m}$, and set $\beta=\max \left\{\alpha \in S \mid H \cap E_{\alpha} \neq \phi\right\}$. Then

$$
\begin{aligned}
\Sigma_{L^{\prime}} g(H) \leqq \mathrm{g}\left(\Sigma_{E}(H)\right) & =g\left(\Sigma_{E_{\beta}}\left(H \cap E_{\beta}\right)\right)=g_{\beta}\left(\Sigma_{E_{\beta}}\left(H \cap E_{\beta}\right)\right) \\
& =\Sigma_{L^{\prime}} g_{\beta}\left(H \cap E_{\beta}\right) \leqq \Sigma_{L^{\prime}} g(H) .
\end{aligned}
$$

So

$$
\Sigma_{L^{\prime}} g(H)=g\left(\Sigma_{E}(H)\right) \text {. }
$$

I wish to thank Dr. A. Horn and the referee for their valuable comments concerning this paper.

\section{References}

1. R. Balbes, Projective and injective distributive lattices, Pacific J. Math. 21 (1967), 405-420.

2. R. Balbes and A. Horn, Order sums of distributive lattices, Pacific J. Math. 21 (1967), 421-435.

3. G. Birkhoff, Lattice theory, Amer. Math. Soc. 25, (1964).

4. C. C. Chang and A. Horn, On the representation of $\alpha$-complete lattices, Fund. Math. 51 (1962), 253-258.

5. P. Crawley, Regular embeddings which preserve lattice structure, Proc. Amer. Math. Soc. 13 (1962), 748-752.

6. R. Sikorski, Boolean Algebras, Academic Press, 1964.

7. - Products of abstract algebras, Fund. Math. 39 (1952).

Received July 10, 1967.

UNIVERSity of Missouri at ST. Louis 



\section{PACIFIC JOURNAL OF MATHEMATICS}

\section{EDITORS}

\section{H. ROYDEN}

Stanford University

Stanford, California

\section{J. Dugundu}

Department of Mathematics University of Southern California Los Angeles, California 90007

RICHARD ARENS

University of California Los Angeles, California 90024

ASSOCIATE EDITORS
E. F. Beckenbach
B. H. NEUMANN
F. WoLF
K. YosidA

\section{SUPPORTING INSTITUTIONS}

\author{
UNIVERSITY OF BRITISH COLUMBIA \\ CALIFORNIA INSTITUTE OF TECHNOLOGY \\ UNIVERSITY OF CALIFORNIA \\ MONTANA STATE UNIVERSITY \\ UNIVERSITY OF NEVADA \\ NEW MEXICO STATE UNIVERSITY \\ OREGON STATE UNIVERSITY \\ UNIVERSITY OF OREGON \\ OSAKA UNIVERSITY \\ UNIVERSITY OF SOUTHERN CALIFORNIA
}

STANFORD UNIVERSITY

UNIVERSITY OF TOKYO

UNIVERSITY OF UTAH

WASHINGTON STATE UNIVERSITY

UNIVERSITY OF WASHINGTON

AMERICAN MATHEMATICAL SOCIETY
CHEVRON RESEARCH CORPORATION
TRW SYSTEMS

AMERICAN MATHEMATICAL SOCIETY

TRW SYSTEMS

NAVAL WEAPONS CENTER

Mathematical papers intended for publication in the Pacific Journal of Mathematics should be in typed form or offset-reproduced, double spaced with large margins. Underline Greek letters in red, German in green, and script in blue. The first paragraph or two must be capable of being used separately as a synopsis of the entire paper. It should not contain references to the bibliography. Manuscripts, in duplicate if possible, may be sent to any one of the four editors. All other communications to the editors should be addressed to the managing editor, Richard Arens, University of California, Los Angeles, California 90024.

Each author of each article receives 50 reprints free of charge; additional copies may be obtained at cost in multiples of 50 .

The Pacific Journal of Mathematics is published monthly. Effective with Volume 16 the price per volume (3 numbers) is $\$ 8.00$; single issues, $\$ 3.00$. Special price for current issues to individual faculty members of supporting institutions and to individual members of the American Mathematical Society: $\$ 4.00$ per volume; single issues $\$ 1.50$. Back numbers are available.

Subscriptions, orders for back numbers, and changes of address should be sent to Pacific Journal of Mathematics, 103 Highland Boulevard, Berkeley 8, California.

Printed at Kokusai Bunken Insatsusha (International Academic Printing Co., Ltd.), 7-17, Fujimi 2-chome, Chiyoda-ku, Tokyo, Japan.

PUBLISHED BY PACIFIC JOURNAL OF MATHEMATICS, A NON-PROFIT CORPORATION

The Supporting Institutions listed above contribute to the cost of publication of this Journal, but they are not owners of publishers and have no responsibility for its content or policies. 


\section{Pacific Journal of Mathematics \\ Vol. 27, No. 3}

March, 1968

Charles A. Akemann, Invariant subspaces of $C(G) \ldots \ldots \ldots \ldots \ldots \ldots . \ldots 41$

Dan Amir and Zvi Ziegler, Generalized convexity cones and their duals ... . 425

Raymond Balbes, On ( $J, M, \mathrm{~m})$-extensions of order sums of distributive

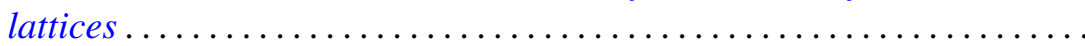

Jan-Erik Björk, Extensions of the maximal ideal space of a function algebra ........................................ 453

Frank Castagna, Sums of automorphisms of a primary abelian group ...... 463

Theodore Seio Chihara, On determinate Hamburger moment problems ..... .

Zeev Ditzian, Convolution transforms whose inversion function has complex

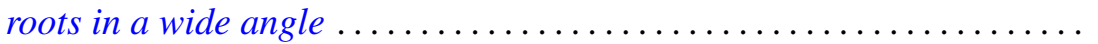

Myron Goldstein, On a paper of Rao .

Velmer B. Headley and Charles Andrew Swanson, Oscillation criteria for

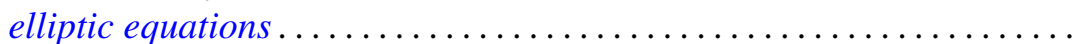

John Willard Heidel, Qualitative behavior of solutions of a third order nonlinear differential equation............................

Alan Carleton Hindmarsh, Pick's conditions and analyticity.............

Bruce Ansgar Jensen and Donald Wright Miller, Commutative semigroups

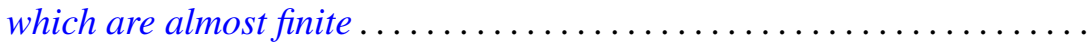

Lynn Clifford Kurtz and Don Harrell Tucker, An extended form of the

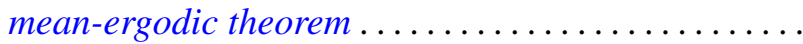

S. P. Lloyd, Feller boundary induced by a transition operator ...

Henry B. Mann, Josephine Mitchell and Lowell Schoenfeld, A new proof of the maximum principle for doubly-harmonic functions ...

Robert Einsohn Mosher, The product formula for the third obstruction ..

Sam Bernard Nadler, Jr., Sequences of contractions and fixed points ...

Eric Albert Nordgren, Invariant subspaces of a direct sum of weighted shifts...

Fred Richman, Thin abelian p-groups ...

Jordan Tobias Rosenbaum, Simultaneous interpolation in $\mathrm{H}_{2}$. II ...

Charles Thomas Scarborough, Minimal Urysohn spaces .

Malcolm Jay Sherman, Disjoint invariant subspaces..... .

Joel John Westman, Harmonic analysis on groupoids....

621

William Jennings Wickless, Quasi-isomorphism and TFM

Minoru Hasegawa, Correction to "On the convergence of resolvents of operators" 